\title{
A Co-opetitive Framework for the Hub Location Problems in Transportation Networks
}

\author{
Rahimeh Neamatian Monemi ${ }^{\mathrm{a}, \mathrm{b}}$, Shahin Gelareh ${ }^{\mathrm{c} *}$, Saïd Hanafi ${ }^{\mathrm{d}}$ and Nelson Maculan ${ }^{\mathrm{e}}$ \\ ${ }^{a}$ Southampton Business School, University of Southampton, Southampton, SO17 1BJ, United Kingdom \\ ${ }^{b}$ Centre for Operational Research, Management Science and Information Systems (CORMSIS), \\ University of Southampton, Southampton, SO17 1BJ, United Kingdom \\ ${ }^{c}$ Portsmouth Business School, University of Portsmouth, UK \\ ${ }^{d}$ LAMIH UMR CNRS 8201 - Université de Valenciennes, 59313 Valenciennes Cedex 9, France \\ ${ }^{e}$ Federal University of Rio de Janeiro, COPPE-PESC, P.O. Box 68511, Rio de Janeiro, RJ 21941-972, \\ Brazil
}

\begin{abstract}
In this paper, we present the first model of co-opetition for a Hub Location Problem (HLP) between two Logistics Service Provider (LSPs) companies where the mother company is the owner of infrastructure. The LSPs would like to cooperate with each other by establishing joint edges with limited capacities connecting their service networks. Such services are in form of pendulum services (a direct service between two points) between nodes of different networks. Additional market can be generated as a result of joining the two networks. At the same time, a competition is taking place between the two operators to increase their share from the additional market generated. In order to solve this problem, we propose a matheuristic approach combining a local search algorithm and a Lagrangian relaxation-based approach. In our matheuristic algorithm, the neighborhood solutions are evaluated using a Lagrangian relaxation-based approach. Numerical results of applying the proposed algorithm on a real case study of the problem are presented.
\end{abstract}

Keywords: co-opetition; cooperation; competition; hub location problem; transportation; Lagrangian heuristic

AMS Subject Classification: 90B10,90C11

\section{Introduction}

Co-opetition (simultaneous pursuit of collaboration and competition) is a neologism coined to describe cooperative competition. In this kind of competition, players cooperate by exchange of information, sharing infrastructure and/or facilities etc. in order to generate additional market for the collaboration as a whole. Simultaneously, the operators compete to increase their own share of the additionally generated market. Ideally, mechanism must be designed in such a way that stability of the system is not harmed by one of the operators trying to do better by considering playing unilaterally and stepping out of the 'alliance'. Furthermore, the effect of cannibalization should be minimized if both operators are subsidiaries of the same company.

The co-opetition combines the advantages of both cooperation [30] and competition [10] for a given company allowing it to increase its profit and affect its surrounding environment. Several fields in management research benefit from

\footnotetext{
*Corresponding author. Email: shahin.gelareh@\{univ-artois.fr;gmail.com $\}$
} 
the co-opetition strategies (e.g. strategic management, organizational studies and experimental economics, see $[4,5,32])$.

In this paper, we consider short term collaboration among a very small number of operators. The objective is actually providing service to a very limited number of additional Origin-Destination (O-D) demands (spot cargos that cannot be accommodated within the current service network) or among a set of players over a longer horizon and a wider geographical zone, on a regular basis. In the earlier case, this is usually achieved by outsourcing the transport service from a competitor to carry out the mission while in the latter case we often refer to the term 'alliance' composed of 'allies' to describe the structure. On one hand, the additionally generated market share is absorbed and on the other hand the partner can improve utilization of its fleet of transporters to exploit better economies of scale. Quite a few examples of the strategic alliances exist in airlines (e.g. Star alliance with allies in five continents), liner shipping alliances (recently launched P3 Alliance, Grand Alliance, New World Alliance etc.) and railway-airline alliances (e.g. Rail\&Fly, an alliance between the DBahn and several airlines from different continents, TGVair, an alliance between the French railway company SNCF and some airlines etc.).

To the best of our knowledge, literature of operations research is not aware of many quantitative models and contributions in the area of co-opetition.

De Ngo and Okura [9] studied the impact of privatization on the degree of cooperation and competition in a mixed oligopoly market that comprises one semipublic firm and one private firm. Each firm is assumed to determine the level of two types of effort. The first one is a cooperative effort made to enlarge the total market size and the second one is a competitive effort made to increase market share. Their results show that the competitive effort level of the semipublic firm is smaller than that of the private firm. The more the semipublic firm is concerned for social welfare, the less it competes. They also show that the semipublic firm expends more cooperative effort than does the private firm.

Gnyawali and Park [17] investigated the situation under which a co-opetition between large firms occurs, evolves, and impacts the participating firms and the industry. They propose a multi-level conceptual framework by combining literaturebased conceptual arguments and insights from an in-depth study of an exemplar case of co-opetition between Samsung Electronics and Sony Corporation.

Zhang and Frazier [33] studied the supply chain partnership with a competitor. They studied dynamic alliance formation among competing firms with a multiperiod model. In each period, there is a two-stage game of co-opetition. In Stage 1, two competing firms decide on forming a partnership by negotiating a contractual agreement; and in Stage 2, all firms in the market engage in price competition.

$\mathrm{Li}$ et al. [20] provided a conceptual model for examining the effects of cooperation and conflicts on a manufacturer's knowledge acquisition process.

Lin and Huang [21] proposed a theoretical framework for characterizing coopetition in international maritime shipping and investigating how carriers can manage their business models.

In the literature, there are only a few works on the competitive hub location problem.

Adler and Smilowitz [2] presented a game theoretic framework to analyze global alliances and mergers in the airline industry under competition. The framework can help airlines identify partners and network structures, and help governments predict changes in social welfare before accepting or rejecting proposed mergers or alliances. 
Martın and Román [25] developed a game theoretic model that analyzes the competition for hub location when airlines operate in deregulated intercontinental markets. The whole process is viewed as a dynamic game of complete information played in two stages: location and competition.

Marianov et al. [24] proposed a competitive hub location problem. The customer capture is sought, which happens whenever the location of a new hub results in a reduction of the current cost (time, distance) needed by the traffic that goes from the specified origin to the specified destination.

Gelareh et al. [14] proposed a mixed integer programming formulation for huband-spoke network design in a competitive environment. This model was based on the model presented in [13]. It addresses the competition between a newcomer liner service provider and an existing dominating operator, both operating on huband-spoke networks. The newcomer company maximizes its market share, which depends on the service time and transportation cost by locating a predefined number of hubs at candidate ports and designing its network.

Lüer-Villagra and Marianov [22] described a situation in which an existing transportation company operates a hub-and-spoke network, and a new company wants to enter into the same market, using an incomplete hub-and-spoke network. The entrant maximizes its profit by choosing the best hub locations and network topology and applying optimal pricing, considering that the existing company applies mill pricing. Customers behavior is modeled using a logit discrete choice model.

Gutiérrez-Jarpa et al. [18] deal with a case of modal competition in rapid transport network design.

Mahmutogullari and Kara [23] proposed a competitive hub location problem where the market is assumed to be a duopoly. Two decision-makers (or firms) sequentially decide locations of their hubs and then customers choose one firm with respect to provided service levels. Each decision-maker aims to maximize his/her own market share. Čvokić et al. [8] deal with a Stackelberg approach where in a Leader and a Follower, are sequentially creating their hub and spoke networks to attract customers in a market where prices have fixed markups. Each competitor wants to maximize his profit, rather than a market share. Demand is split according to the logit model and their goal is to find the optimal hub and spoke topology for the Leader.

Adler [1] evaluated airline profit based on micro-economic theory of behaviour under deregulation. Through a two-stage Nash best-response game, equilibria in the air transportation industry is sought to evaluate the most profitable HS network for an airline to survive in a deregulated environment.

In hub location under competitive settings one can refer to [29] for a Stackelberg hub location problem wherein a big firm (leader) competes with several smaller ones (followers) to maximize their own profit while there is no competition among the smaller firms. In Sasaki [27], the leader locates $p$ hubs first and the followers locate $q$. To avoid the possibility of unprofitable services, they incorporate flow threshold constraints into their bi-level programming model which is solved by enumeration. Sasaki et al. [28] deal with hub-arc location problem. Eiselt and Marianov [11] presents a $p$-hub location model wherein customers have gravitylike utility functions. Their models is a probabilistic one wherein customers choose an airline depending on a combination of functions of flying time and fare. The (conditional) follower's hub location problem is solved by means of a heuristic concentration method.

Interested readers are also referred to the recent Hub Location Problems (HLPs) literature review in Alumur and Kara [3] and [7] as well as other contributions by 
This work is motivated by a real case study from the liner shipping industry. A holding company that previously owned a single liner shipping company (a regional operator) decided to acquire a second one that operates on a different region. These two liner shipping companies, have an almost zero (very minimal) market overlap with each other. The holding wants to study the viability and potential gains that it can expect if it unifies the networks and establishes different services among them. Due to the complexity of considering different service types, in this study, we only concentrate on pendulum services. A pendulum service is a shuttle service between two points, $p_{1} \rightarrow p_{2} \rightarrow p_{1}$.

In this work, we are dealing with a duopoly market; a co-opetition that is taking place between two sister companies belonging to the same mother company to increase their share from the generated additional market. In Section 2, a detailed problem description is presented. In Section 3, we first present the mathematical model, in its generic form, for the co-opetition in a hub-and-spoke network design. We then give further details for an application in liner shipping. A matheuristic approach to solve the problem is proposed in Section 4. Our numerical experiments are presented and discussed in Section 5 and finally in Section 6, we conclude our work and highlight some further research directions for the future studies.

\section{Problem Description}

There are two Logistics Service Providers (LSPs) denoted by $\mathcal{A}$ and $\mathcal{B}$ of the same mode of transport operating in different regions (perhaps with minimal or negligible interconnections). These two LSPs are not in a direct competition and/or collaboration as the local regulations do not allow one to operate on the entire regional network of another one (e.g. as a result of Convention on Internal Civil Aviation -Chicago convention- or the shipping regulation in the territorial waters of some countries mainly in Asia region that make it, to a large extent, reserved for domestic players (sometimes for the same operator, only a part of its fleet complying with certain regulations concerning flagging, maintenance history, crew background etc. is authorized to operate in certain regions, resulting in a disconnectedness between the networks over which its fleet is operating.). The set of clients that can be potentially served by an LSP $o$ is represented by $\mathcal{N}_{o}$. An LSP $o \in \mathcal{O}=\{\mathcal{A}, \mathcal{B}\}$ operates on a hub-and-spoke structure $\mathcal{N}_{o}=\mathcal{N}_{o}^{H} \cup \mathcal{N}_{o}^{S}$, where $\mathcal{N}_{o}^{H}$ is the set of hub nodes of operator $o$ and $\mathcal{N}_{o}^{S}$ represents the set of spoke nodes of operator $o$.

Here, every spoke in $\mathcal{N}_{o}^{S}$ is allocated to one or more hub nodes in the set of hub node $\mathcal{N}_{o}^{H}$ (see Figure 1). In Figure 1, the rectangular objects represent hub nodes and the circles represent spoke nodes. The solid lines represent the hub-level edges and the dashed lines are the spoke edges connecting spoke nodes to hub nodes. Note that there is no direct connection between two spoke nodes. The lighter left-hand side nodes belong to the first operator and the darker right-hand side nodes belong to the second operator.

In such a network, for every LSP $o \in \mathcal{O}$, the O-D flows chosen to be served will follow a path passing through at least one element of the hub-level network. For a given LSP, once an O-D flow leaves its origin and arrives to the hub-level network, it traverses the hub-level network either up until the destination hub 


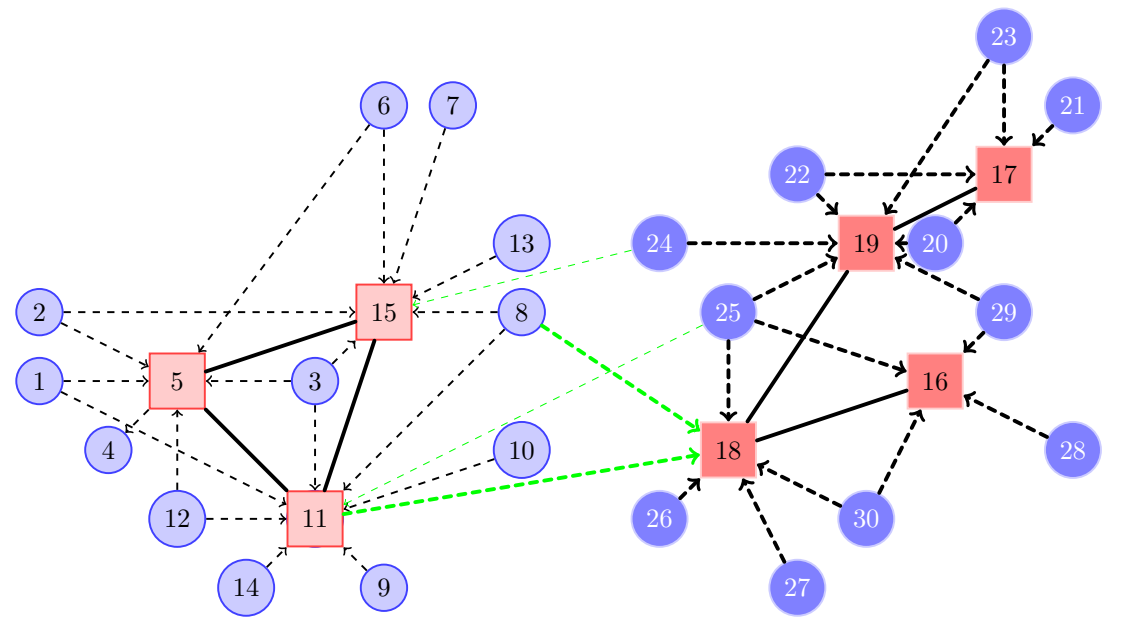

Figure 1.: An example of a Hub-and-Spoke co-opetition network.

node or a hub node where the destination spoke node is allocated to it. Therefore, the hub-level network is a connected graph. However, the joint hub-level network (union of the hub-level networks of LSPs) is not initially a connected network and is composed of exactly two components. In other words, the LSPs do not share any hub node/edge (see Figure 2).

Let us denote cardinality of a set $A$ by $\operatorname{card}(A)$ (equivalently, $|A|$ ). In general,

$$
\mathcal{N}_{\mathcal{A}} \cap \mathcal{N}_{\mathcal{B}} \neq \emptyset \quad \text { while } \quad \operatorname{card}\left(\mathcal{N}_{\mathcal{A}} \cap \mathcal{N}_{\mathcal{B}}\right)<<\min \left\{\operatorname{card}\left(\mathcal{N}_{\mathcal{A}}\right), \operatorname{card}\left(\mathcal{N}_{\mathcal{B}}\right)\right\}
$$

i.e. the LSPs' networks may still share some nodes. As shown in Figure 1, a spoke node in one LSP's network can be also a spoke in another LSP's network and therefore allocated to one or more hub nodes in each one of the two (see nodes \#8, \#24 and \#25). Such allocations to two different networks are not to diversify the market for the same product, rather this is about different products exclusively supplied by (or demanded from) one of the networks. Furthermore, an LSP may use a node as a hub node while other LSP consider it as a spoke node (see node \#11, which is a spoke node of the right-hand side LSP.), yet, the edge \#11 - \#18 is not recognized as a hub edge because none of the two LSPs recognizes the both end-points as hub nodes.

As the networks of the LSPs are designed independently, such common spoke nodes are identified a posteriori and do not play any role in transferring flows originating from all or some origins in one network to all or some destinations in another one. They are only representing the flow emanating from and destined to themselves with respect to every network, independently. Therefore, at the initial phase, as there is no interaction between the LSPs' networks, these common nodes do not have any particular functionality and one can make a copy of such nodes and create one graph per LSP with no edge connecting the two sub-graphs resulting in two separated graph components.

In a competitive environment wherein LSPs $\mathcal{A}$ and $\mathcal{B}$ are operating and competing to occupy more capacity from the jointly established service for their clients in each other's networks, a service level (a so-called 'market transit time' in this context) plays the key role in allocation of market shares to an 

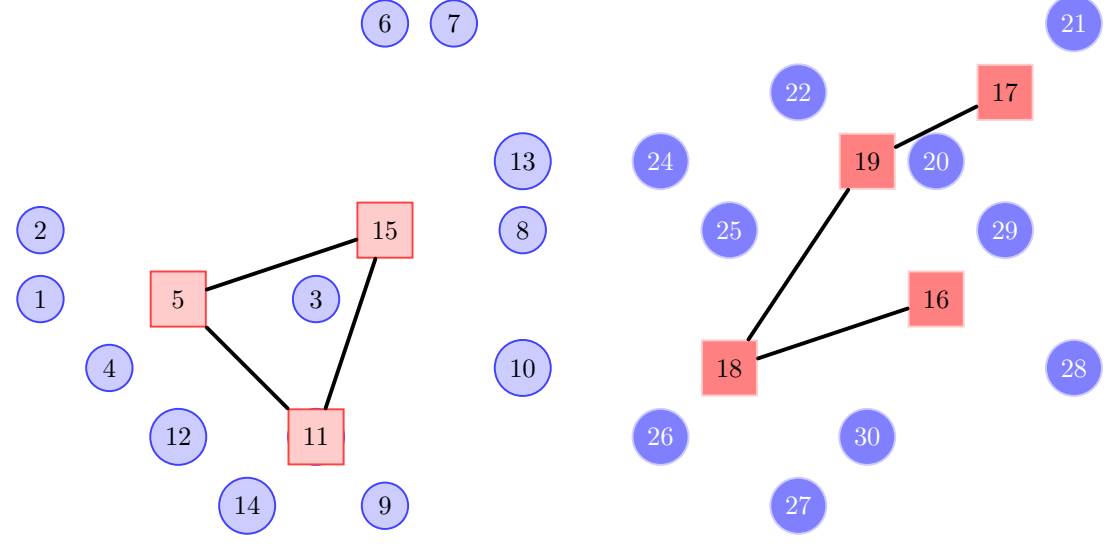

Figure 2.: In the initial configuration, no hub edge connects any pair of hub nodes of different LSPs.

LSP. In an application such as long-haul liner shipping, as a rule of thumb, if one LSP cannot provide a transit time of at most $\gamma$ percent above the direct point-to-point transit time (the usual market transit time that other players can offer for the same O-D pair) then it certainly cannot absorb any part of the market.

We call a given O-D demand captured, if it is an internal demand of a given operator and is already served before co-opetition being launched. The flows to be captured from non-captured O-D pairs are unsplittable.

Remark that, in terms of routing flows, no direct spoke-to-spoke connection is allowed; neither in the internal network of each operator nor in the co-opetition network (the network resulted after joining different operators).

An LSP is not authorized to capture O-D demands of the original network of another LSP. For an LSP, the new market includes only those demands whose origins were in its own original network and their destinations were in the original network of the other LSP (and vice versa). As a consequence, the competition can only be realized on the collaboratively established edges (between different LSPs). It must be noted that in a given feasible solution, even if the collaboration level of an operator is zero, it can still have a positive competition level on those edges because even if investment for installing hub edges is not profitable for it, still the fact that it allows other to gain market on its territory must have some rewards for it.

The problem is described as follows: Every node in the LSP $\mathcal{A}$ can potentially be a client for the LSP $\mathcal{B}$ and vice versa. We seek a unified hub-level network, which is a connected graph and in which every LSP can potentially gain more if operating on the new unified system compared to its own initial network. That is, by possibly restructuring its own network composed of hub nodes, spoke nodes and their allocations, the total revenue is at least as good as before, for every operator. We assume that all the existing infrastructure of every LSP can be still operational in the unified network. As a result, the O-D transit times are never increased although may even be reduced (this can occur because the additional profit generated by the increased market share of inter-network demands can be 
invested in installing new hub nodes and edges for every operator and as a result new paths with shorter transit times can be identified). Such reduced transit times on one hand can help in capturing additional internal demand market and on the other hand the reduced transit times for internal demands may be interpreted as lower transportation costs if the same shipping setting is kept.

If any of the existing hub edges of operators appear in the unified hub-level network, no cost will be incurred for reinstalling such a facility and no capacity is considered for such hub edges. The existing infrastructures will be available to both LSPs with no additional charge. Any new hub edge connecting hub nodes of different LSPs is 'collaboratively installed and competitively exploited'. An LSP is responsible to pay the additional costs of installing a new hub node and hub edge among its own nodes. The cost of installing a new hub edge between two service providers is shared between them.

A normalized factor/level of cooperations influences the contribution of each LSP in the fixed cost of installation. Another normalized factor/level of competition determines the type of transporter (i.e. capacity and possibly other features) deployed on the jointly installed hub edges (a larger transporter that offers better economies of scale competes better on both the transportation cost and capacity).

For every LSP, the cost of collaboration, which accounts for the contribution in installing new hub edges and hub nodes, has a quadratic form (if one does not contribute the other one contributes by $100 \%$ and partial contributions by both parties reduce the overall cost of a jointly installed service), and the competition cost that is resulted by deploying a given transporter, with a given design speed and capacity, has a linear form. The objective is to maximize their profits that account for the revenue after deducting hub facility installation, transportation, competition and cooperation costs.

\section{Mathematical Formulation and Maritime Transport Case}

In this section, we first present the model in general form and then develop the cost components for the special case of maritime transport.

\section{1. $\quad$ Mixed integer programming formulation}

We assume that in the initial state, every Liner Shipping Company (LSC) is operating on its own network, i.e. everyone with its existing edges. Moreover, as both LSCs are subsidiaries of the same company, in this work the focus and outcome of co-opetition will be on the maximization of the total profitability of the joint cooperation/competition rather than finding an equilibrium between them. While the latter can be modeled in form of a game and solved by a diagonalization process (through which the operators exchange information while each one tries to maximize its own profit) or similar methods, the former is the focus of this work and can be modeled using a single mathematical program.

As mentioned previously, the cooperation is modelled by participation of every operator in installing new connections (with their end-points in the networks of 
different operators) and deploying its fleet composed of transporters of different classes operating on different speeds on them. In the course of competition, the operators are allowed to install new connections (hub edges) within their own/initial network where both end-points belong to the same operator (we call them internal edges) at their own costs while an edge connecting two nodes from two different operators must be installed cooperatively (even with an operator having a level of cooperation equivalent to zero on some particular edges). Every operator is allowed to operate its own transporter $v$ (vessel, vehicles etc.) on a collaboratively installed edge (even if it did not contribute at all in installing that edge). In some applications the cost of deploying such transporters depend heavily on the operational (market) speed. Normally, such speeds are limited to a set of discrete values.

In the co-opetitively designed network, if an inter-network O-D demand (demands with end-points belonging to different LSCs) is captured, the path will be composed of three segments: 1) from origin in the LSCo to an end-point of a collaboratively established edge belonging to it, 2) transportation on the collaboratively established edge, and 3) the path from the other end-point of the collaboratively established edge to the destination between nodes of second operator. This includes two transshipments taking place when changing the route segments. In this work, to avoid further complexity in the model, we do not take into account these two transshipment explicitly but it is implicitly considered (to be independent of volume of flow) when talking about cost of installing an edge.

For every operator $o \in \mathcal{O}$, the parameters and decision variables are introduced in Table 1 and Table 2 , respectively.

The level of collaboration is defined as a normalized factor representing the relative effort (e.g. monetary contribution) of an operator to the total efforts (e.g. whole investment) involved in collaboratively realizing a project (e.g. establishing a pendulum service - an edge). Such a collaboration aims at generating added value (e.g. additional market share) that is then subject to a competition between operators. The level of competition is defined as a normalized factor representing how operators intensify (relative to each other) their efforts in absorbing their share from the aforementioned added values.

The co-opetition cost for the operator $o$ has the following form [9]:

$$
z_{o}=(r-c) y \frac{x_{o}}{x_{A}+x_{B}}-k_{x} x_{o}-k_{y} y_{o}^{2},
$$

where $r$ is the revenue, $c$ is the cost, $x_{o}$ is the competitive effort level of operator $o, y$ is the decision to be made and $k_{x} x_{o}\left(k_{y} y_{o}^{2}\right)$ represents the cost of expending competitive (cooperative) efforts, respectively (see Section 3.2 for further details).

Cooperation cost: The cooperation cost function is a function that measures the cost of contribution of each LSC to install the jointly exploited hub edges (edges with end-points in different LSCs). Such a function, according to [9], is a quadratic function. We refer to the cooperation function by $f\left(z_{k l}^{+}\right)$and will further elaborate on its structure in Section 3.2. 


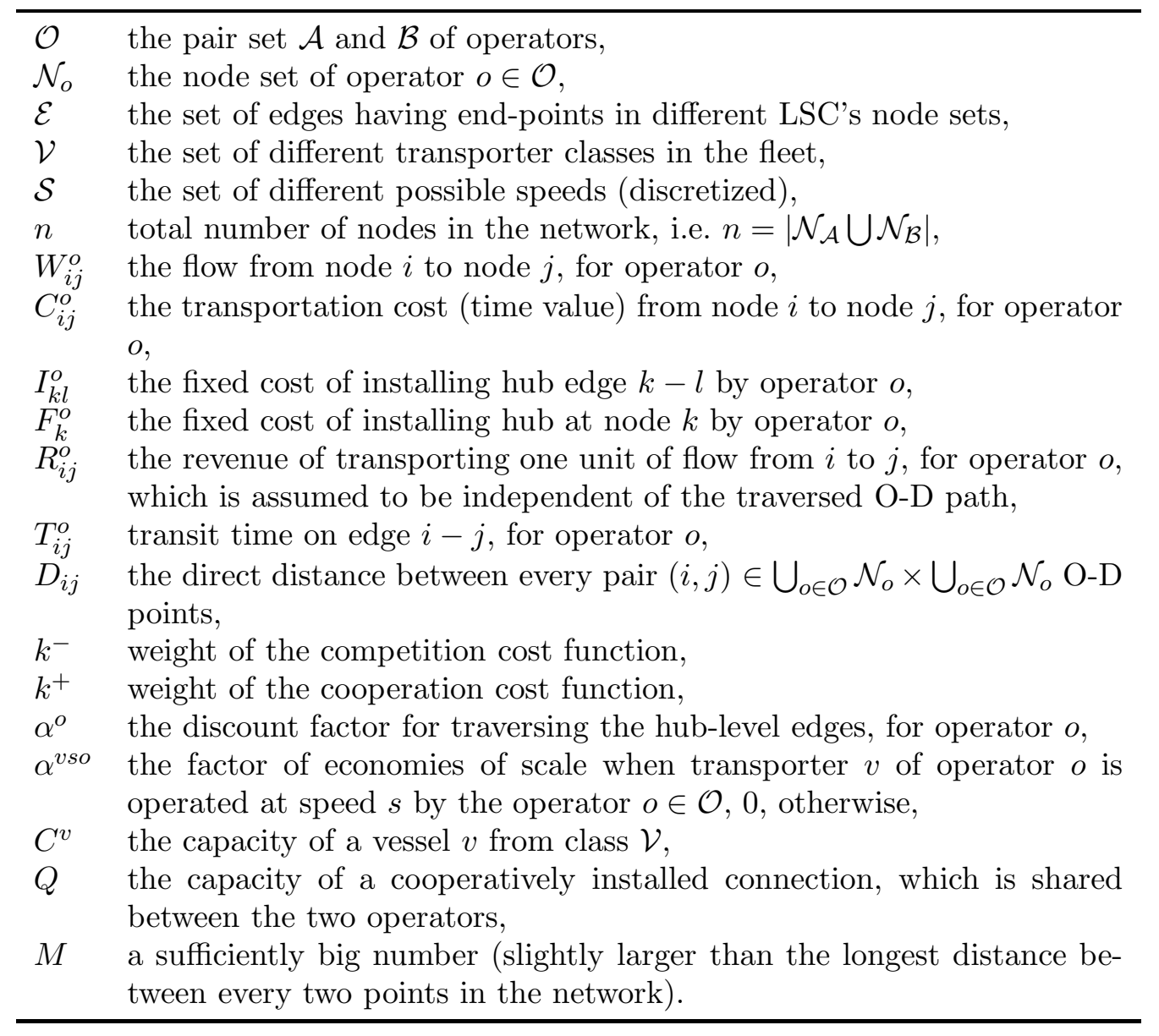

Competition cost: The LSCs are competing with each other to claim capacity on the jointly exploited hub edges and transporting as much as possible of the captured inter-network O-D demands. Such a competition arises from difference sources. The prerequisite is that the market is captured with respect to a transportation time that does not go beyond the market transit time, i.e. $(1+\gamma) T_{i j}^{o}$. For an operator, this is either automatically fulfilled by the structure of (hub-level) network or it can be done by increasing the sailing/driving speed on the jointly operated hub edges. On the other hand, if this goal can be achieved only by increasing the speed and increasing the bill of bunker fuel (bunker fuel represent any type of fuel oil used aboard vessels and the the corresponding cost is referred to as the bunker bill), then the competition is taking place by occupying as much of volume of the jointly operated hub edge as possible to maintain a profitable business coping with the increase in the bunker bill. The latter is done by deploying larger transporters offering better economies of scale (lower transportation cost per unit of load). Such an increase in the volume translates to a reduction in the capacity remaining for the other LSC. The third scenario is that there would be a sufficient slack between the transit time of LSC's O-D path and $(1+\gamma) T_{i j}^{o}$ allowing it to have some market share even if the LSC reduces its sailing speed and sails in Slow-steaming mode. We refer to the competition function by $f\left(z_{k l}^{-}\right)$and will further elaborate on its structure in Section 3.2.

Let $\mathcal{N}_{o}^{0}, \mathcal{E}_{o}$ and $\mathcal{E}_{o}^{1}$ be the set of existing hub nodes, potential edges and hub 


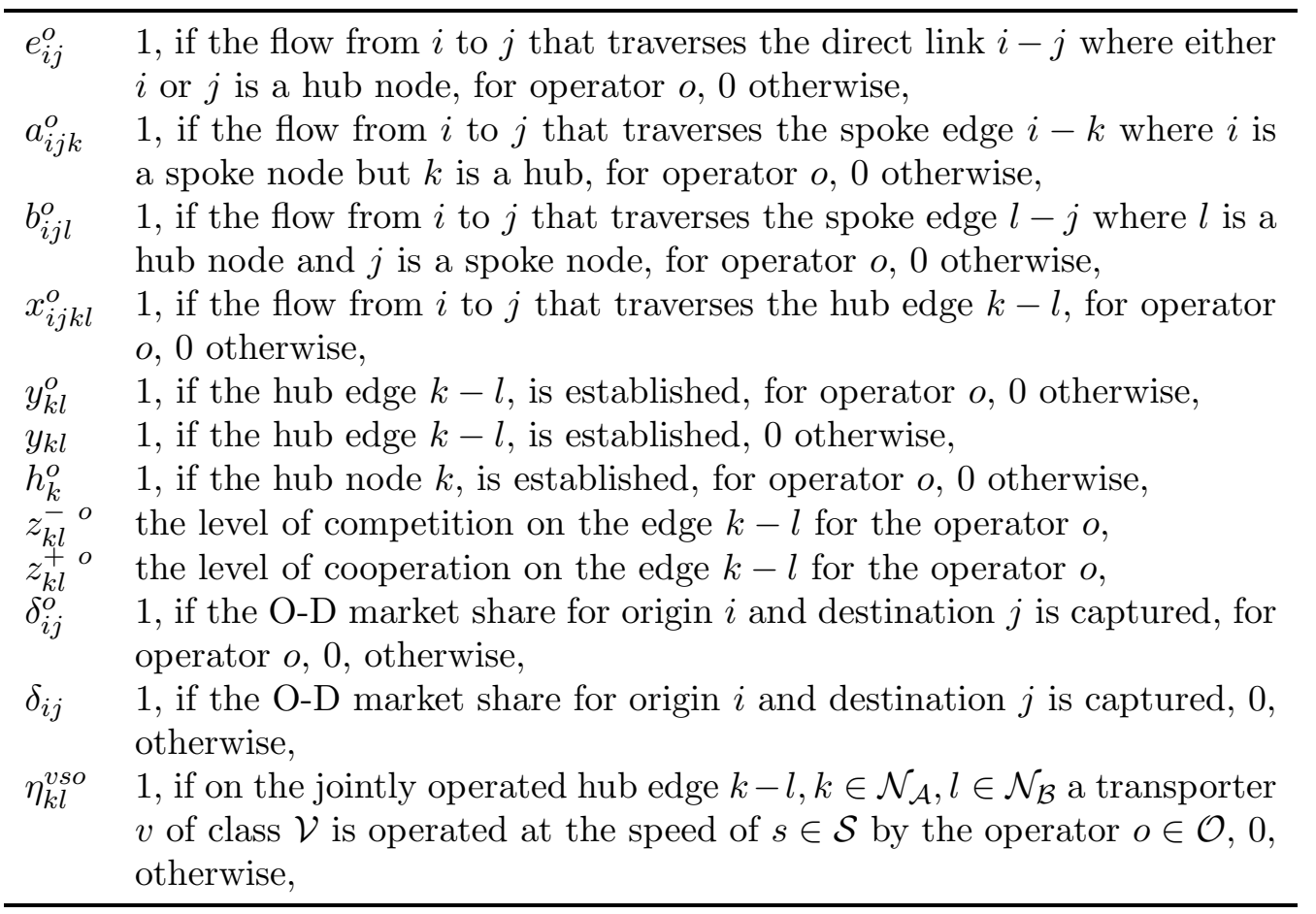

edges of LSCo, respectively, at the initial state. Let $\bar{o}=\mathcal{O}-\{o\}$, i.e. if $o=\mathcal{A}$ then $\bar{o}=\mathcal{B}$ and vice versa. Moreover, let $\mathcal{I}_{o}^{0}$ be the set of all O-D pairs (origins and destinations inside $\mathcal{N}_{o}$ ) for operator $o$ for which the market was not captured in the initial state. Let us also define $\mathcal{J}=\left\{\{i, j\} \mid j \neq i,(i, j) \in \mathcal{I}_{o}^{0} \cup\left(\mathcal{N}_{o} \times \mathcal{N}_{\bar{o}}\right), \forall o \in \mathcal{O}\right\}$ and $\mathcal{E}=\left\{\left(\mathcal{N}_{\mathcal{A}} \times \mathcal{N}_{\mathcal{B}}\right) \vee\left(\mathcal{N}_{\mathcal{B}} \times \mathcal{N}_{\mathcal{A}}\right)\right\}$

The mathematical model for the Hub Location Problems in a Duopoly Co-opetitive Environments (COOPTV-HLP) follows:

$$
\begin{aligned}
\max & \sum_{o \in \mathcal{O}}\left(\sum_{i, j \neq i} R_{i j}^{o} \delta_{i j}^{o}-\left[\sum_{k, l \neq k:(k, l) \notin \mathcal{E}} \alpha^{o} C_{k l}^{o} x_{i j k l}^{o}+\right.\right. \\
& \sum_{(k, l) \in \mathcal{E}, k \neq l} \alpha_{k l}^{v s o} C_{k l}^{o} \eta_{k l}^{v s o} x_{i j k l}^{o} \\
& \left.+\sum_{k \neq i, j} C_{i k}^{o} a_{i j k}^{o}+\sum_{k \neq i, j} C_{k j}^{o} b_{i j k}^{o}+C_{i j}^{o} e_{i j}^{o}\right] \\
& \left.-\sum_{k \in \mathcal{N}_{o}-\mathcal{N}_{o}^{o}} F_{k}^{o} h_{k}^{o}-\sum_{(k, l) \in\left(\mathcal{N}_{o} \times \mathcal{N}_{o}\right)-\mathcal{E}_{o}^{1}} I_{k l}^{o} y_{k l}^{o}\right) \\
& +\sum_{(k, l) \in \mathcal{E}}-f\left(z_{k l}^{-o}\right)-\sum_{(k, l) \in \mathcal{E}} f\left(z_{k l}^{+o}\right)
\end{aligned}
$$

s.t. 
$\sum_{o \in \mathcal{O}} z_{k l}^{+o}=y_{k l}$,

$\forall(k, l) \in \mathcal{E}$,

$\sum_{o \in \mathcal{O}} z_{k l}^{-o}=y_{k l}$,

$\forall(k, l) \in \mathcal{E}$,

$y_{k l}^{o} \leq h_{k}^{o}, \quad y_{k l}^{o} \leq h_{l}^{o}$,

$\forall o \in \mathcal{O},(k, l) \in \mathcal{E}_{o}-\mathcal{E}_{o}^{1}$,

$y_{k l} \leq h_{k}^{o}, \quad y_{k l}^{o} \leq h_{l}^{\bar{o}}$,

$\forall o \in \mathcal{O},(k, l) \in \mathcal{E}$,

$x_{i j k l}^{o}+x_{i j l k}^{o} \leq \sum_{o^{\prime} \in \mathcal{O}} y_{k l}^{o^{\prime}}$,

$\forall o \in \mathcal{O},(i, j) \in \mathcal{J}, k<l$,

$\sum_{o \in \mathcal{O}}\left(\sum_{l \neq i} x_{i j i l}^{o}+\sum_{l \neq i, j} a_{i j l}^{o}+e_{i j}^{o}\right)=\delta_{i j}$

$\sum_{o \in \mathcal{O}}\left(\sum_{l \neq j} x_{i j l j}^{o}+\sum_{l \neq i, j} b_{i j l}^{o}+e_{i j}^{o}\right)=\delta_{i j}$

$\forall(i, j) \in \mathcal{J}, i \neq j$,

$\sum_{l \neq k, i} x_{i j k l}^{o}+b_{i j k}^{o}=\sum_{l \neq k, j} x_{i j l k}^{o}+a_{i j k}^{o}$,

$\forall o \in \mathcal{O},(i, j) \in \mathcal{J}, k \notin\{i, j\}$,

$\delta_{i j}=\delta_{i j}^{o}$,

$\forall o \in \mathcal{O}, i, j \in \mathcal{N}_{o}, i \neq j$,

$\sum_{o \in \mathcal{O}} \delta_{i j}^{o}=\delta_{i j}$

$\forall(i, j) \in \mathcal{E}$,

$\sum_{l \neq k} x_{k j k l}^{o} \leq \sum_{o^{\prime} \in \mathcal{O}} h_{k}^{o^{\prime}}$

$\forall o \in \mathcal{O},(k, j) \in \mathcal{J}$,

$\sum_{k \neq l} x_{i l k l}^{o} \leq \sum_{o^{\prime} \in \mathcal{O}} h_{l}^{o^{\prime}}$

$\forall o \in \mathcal{O},(i, l) \in \mathcal{J}$,

$a_{i j k}^{o}+\sum_{l \neq j, k} x_{i j l k}^{o} \leq \sum_{o^{\prime} \in \mathcal{O}} h_{k}^{o^{\prime}}$,

$\forall o \in \mathcal{O},(i, j) \in \mathcal{J}, k \notin\{i, j\}$,

$b_{i j k}^{o}+\sum_{l \neq k, i} x_{i j k l}^{o} \leq \sum_{o^{\prime} \in \mathcal{O}} h_{k}^{o^{\prime}}$,

$\forall o \in \mathcal{O},(i, j) \in \mathcal{J}, k \notin\{i, j\}$, 


$$
e_{i j}^{o}+2 x_{i j i j}^{o}+\sum_{l \neq j, i} x_{i j i l}^{o}+\sum_{l \neq i, j} x_{i j l j}^{o} \leq \sum_{o^{\prime} \in \mathcal{O}}\left(h_{i}^{o^{\prime}}+h_{j}^{o^{\prime}}\right), \quad \forall o \in \mathcal{O},(i, j) \in \mathcal{J},
$$

$$
\begin{array}{ll}
\sum_{k, l \neq k} T_{k l}^{o} x_{i j k l}^{o}+\sum_{k, l \neq k} \frac{D_{k l}}{s} \eta_{k l}^{v s o} x_{i j k l}^{o}+\sum_{k \neq i, j} T_{i k}^{o} a_{i j k}^{o} & \\
+\sum_{k \neq i, j} T_{k j}^{o} b_{i j k}^{o}+T_{i j}^{o} e_{i j}^{o} & \\
\leq(1+\gamma) T_{i j}^{o} \delta_{i j}+M\left(1-\delta_{i j}\right), & \forall o \in \mathcal{O},(i, j) \in \mathcal{J}, \\
x_{i j}^{o} \leq 2-\left(h_{i}^{o}+\sum_{o^{\prime} \in \mathcal{O}} y_{j}^{o^{\prime}}\right), & \forall o \in \mathcal{O},(i, j) \in \mathcal{I}_{o}^{0},
\end{array}
$$

$$
a_{i j k}^{o} \leq 1-h_{i}^{o},
$$$$
\forall o \in \mathcal{O}, i, j \in \mathcal{J}, k \notin\{i, j\} \text {, }
$$

$$
b_{i j l}^{o} \leq 1-\sum_{o^{\prime} \in \mathcal{O}} h_{j}^{o^{\prime}}
$$

$\sum_{o \in \mathcal{O}} \sum_{(i, j) \in \mathcal{J}} W_{i j}^{0} x_{i j k l}^{o} \leq \sum_{o \in \mathcal{O}} \sum_{v \in \mathcal{V}} \sum_{s \in \mathcal{S}} C^{v} \eta_{k l}^{v s o}$

$\forall(k, l) \in \mathcal{E}$,

$$
\begin{aligned}
& \sum_{v, s} \eta_{k l}^{v s o} \leq y_{k l}, \\
& \sum_{v, s, o} C^{v} \eta_{k l}^{v s o} \leq Q \\
& z_{k l}^{-o} \leq \sum_{v, s} \eta_{k l}^{v s o}, \\
& x_{i j k l}^{o}, a_{i j k}^{o}, b_{i j k}^{o}, e_{i j}^{o} \in\{0,1\}, \\
& y_{k l}^{o}, y_{k}^{o}, u_{i j}^{o} \in\{0,1\}, \\
& z_{k l}^{-o}, z_{k l}^{+o} \in[0,1],
\end{aligned}
$$

$\forall o \in \mathcal{O},(k, l) \in \mathcal{E}$

$\forall(k, l) \in \mathcal{E}$,

$\forall o \in \mathcal{O},(k, l) \in \mathcal{E}$,

Objective function (3) is to maximize the profit, i.e., the difference between the revenue and the cost, comprised of the total transportation, facility (hub nodes and hub edges) installation, competition, cooperation and fleet deployment cost (choice of capacity and sailing speed).

Constraints (4)-(5) normalize the cooperation and competition levels, respectively. For a given hub edge $k-l$, if opened, the total cooperation (competition) level, sum up to 1 , and 0 , if the hub edge $k-l$ is not established.

Constraints (6) ensure that for installing a hub edge between nodes of LSCo, 
both end-nodes must be the hubs of the same LSCo. On the other hand, a hub edge $k-l$ connecting nodes of two different operators must have $k$ as a hub node of the LSCo and $l$ as a hub node of the other one (i.e. LSC $\bar{o}$ ). This is guaranteed in (7).

Constraints (8) makes sure that any hub-level flow must traverse the hub edges of operators. Constraints (9)-(11) are the flow constraint taking into account that only one LSC is the winner for one given O-D demand. These constraints on one hand, identify the new O-D flows that LSCo can capture (those intra-network O-D demands that were not initially captured) within its own region $\left((i, j) \in \mathcal{N}_{o} \times \mathcal{N}_{o}\right)$ and on the other hand, the O-D flows with origin in the region of LSCo and destination in the region of other LSC.

Constraints (12) make sure that the non-captured internal demands of an operator are reserved uniquely for itself, if it can capture it. For O-D demands with origin and destination belonging to different operators (intra-network demands), only one of the two can be the winner, if any at all, as in constraints (13).

The constraints (14)-(15) state that for the operator $o$, if the origin is a hub node, it must be a hub node of LSCo but if the destination is a hub node then it can be a hub node of any of the two operators. An intermediate (neither origin nor destination) hub node can be a hub of any of the two operators' regional networks. Constraints (16)-(17) stand for this. For every O-D, the choice of path depends on the status of origin and destination, whether both, neither, or only one of them is a hub node. Constraints (18) ensure this.

By adding constraints (19) we make sure that either by an appropriate choice of speed, we will be able to capture the market or the market is totally lost (big-M constraints). If for a given O-D pair, the transit time is already below $(1+\gamma) T_{i j}^{o}$, one can choose to run into slow-steaming mode and still reduce the fuel consumption while stretching out the transit time to approach the threshold $(1+\gamma) T_{i j}^{o}$.

Constraint (20)-(22) guarantee the correspondence between the definition of variables and the status of nodes. In $a_{i j k}^{o}\left(b_{i j k}^{o}\right)$, node $i$ (node $j$ ) cannot be a hub node and in $e_{i j}^{o}$ at most on of $i$ or $j$ can be a hub node.

In constraint (23), the capacity of every jointly operated hub edge (the sum of capacities of vessels deployed by both operators) is verified. Constraints (24) ensure that an operator chooses a unique state of operation (vessel type, speed and capacity) on a cooperatively installed connection. Constraints (25) ensure that the sum of the capacities deployed by different operators $\left(C^{v}\right)$ on the same edge is bounded by the edge capacity, $Q$. Finally, an operator competes with another one on a leg $k-l$ only if it chooses to deploy a capacity on that connection, as stated in constraints (26).

\subsection{The case of maritime transport}

We present our cooperation and competition cost functions, based on some empirical data collected from the industry.

As mentioned earlier, the cost function of cooperation has a quadratic form [9]. Our empirical studies and data mining on a large dataset from our industrial 


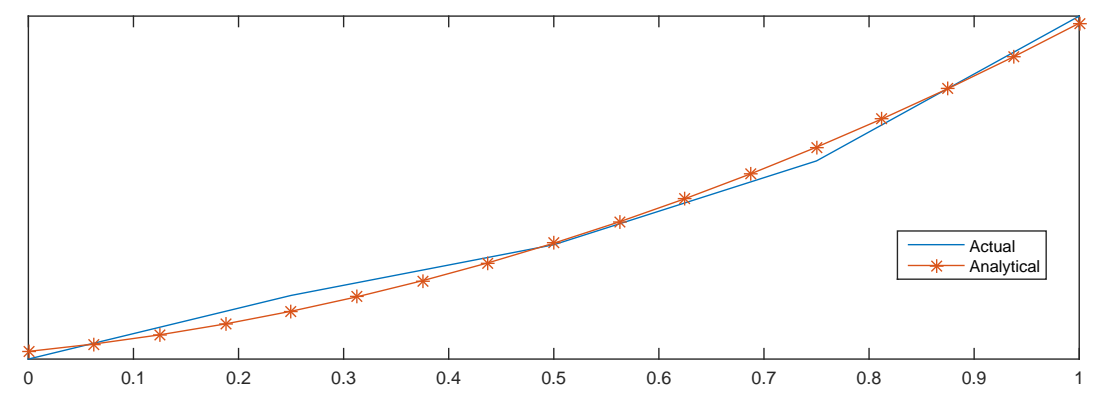

Figure 3.: The real form of a cooperation cost function is shown in solid (blue) line. The quadratic approximation of the real curve is plotted in (red) line-star style. In practice, the approximation error is negligible.

partners reveal that such a function for the operator $o$ can be approximated by the following quadratic form (see Figure 3):

$$
f_{k, l}^{+o}\left(z_{k l}^{+}\right)=\frac{4}{7} I_{k l}^{o}\left(z_{k l}^{+o}\right)^{2}+\frac{68}{175} I_{k l}^{o} z_{k l}^{+o}
$$

This is the cost incurred based on the level of cooperation between two liner service providers. In the absence of any kind of cooperation, no cost is incurred while a full cooperation (being the only investor in establishing connection) incurs the full cost of installation.

According to [26], in liner shipping industry, the total fuel consumption at sea for the main engine $m$ in grams/day $d$ and at a given design speed $s_{0}$ can be estimated as:

$$
F C_{m d}\left(s_{0}\right)=3.775 \times e^{1.996} \times T E U^{1.013} .
$$

In our work, the competition cost is composed of two parts. The first part is interpreted as the impact of the increase/decrease in vessel speed from the initial economic design speed $s_{0}$ (which corresponds to a coefficient $\alpha$ ) to a speed $s$ [31], and the second part is the cost incurred by deploying the vessel for claiming more slots from the whole capacity of the jointly-operated edges. When dealing with containerships, the fuel consumption at the speed $s$ with design speed $s_{0}$, can be calculated using the following cubic function:

$$
F C_{m d}^{v}(s)=F C_{m d}\left(s_{0}\right)\left(\frac{s}{s_{0}}\right)^{3.3}, \quad \forall v \in \mathcal{V}
$$

However, as one can see in Figure 4, on a given interval, this function can be approximated by a straight line (i.e. $a_{s} x_{x}+b_{s}$ ). The approximation becomes even more precise for smaller vessels. As a results, the linear term is substituted as follows:

$$
F C_{m d}^{v}(s)=a_{v} s+b_{v}, \quad \forall v \in \mathcal{V} .
$$

The corresponding $a_{v}$ and $b_{v}$ are reported in Figure 4. However, to avoid 


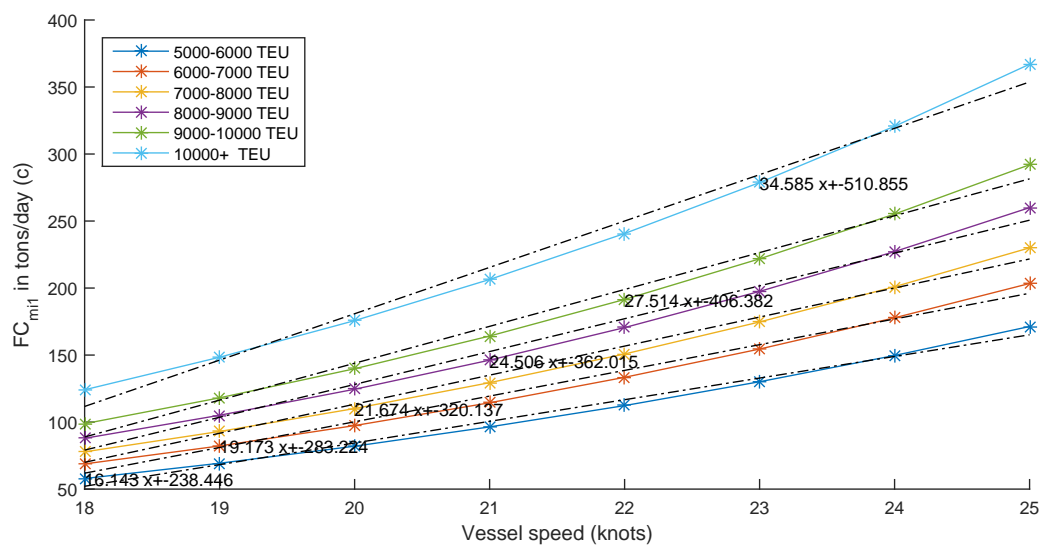

Figure 4.: Fuel consumption as a function of vessel speed for every vessel class is depicted in this figure. The vessels are categorized in 6 classes. For each class, the dashed (black) line represent linear approximations of the corresponding real (colored) curve. In practice, the error in this approximation is rather negligible because every vessel class has its own and very tight interval of speed within which the operators prefer sail. Within such an interval the approximation is almost precise.

introducing further nonlinearity in the model, one can limit oneself to discrete values from among all possible values (e.g. the star-like points on Figure 4).

Let $F C^{v s o}$ be the cost of operating vessel $v$ at speed $s$ for operator $o$, the first part of competition cost follows:

$$
F C_{k l}^{s v}=F C_{m d}^{v}(s) \eta_{k l}^{v s o} \frac{D_{k l}}{24 s}, \quad \forall(k, l) \in \mathcal{E}, s \in \mathcal{S}, v \in \mathcal{V}
$$

If slow-steaming strategy is adopted, it reduces the transportation cost while if a strategy based on increasing speed on the given edge is adopted allowing the total transit time of an O-D pair be below by $(1+\gamma) T_{i j}^{o}$, the transportation cost may increase but at the same time the operator can compete for capturing market share.

The competition cost function for operator $o$ can be approximated by:

$$
f_{k, l}^{-o}\left(z_{k l}^{-}\right)=\sum_{v \in \mathcal{V}, s \in \mathcal{S}} \frac{F C_{k l}^{s v}}{F C_{k l}^{s_{0} v}} z_{k l}^{-o}, \quad \forall(k, l) \in \mathcal{E} .
$$

\section{A Heuristic Approach for the Problem}

Even very small instances of (COOPTV-HLP) (in terms of network and fleet size) are very complex and represent large scale nonlinear programs. We already examined different approaches to solve the model; e.g. directly applying a nonlinear mixed integer programming solver, applying some parameterized linear programs, using some specialized convex approximation methods and also decomposition approaches based on the Generalized Benders Decomposition [16]. All such methods even with a very efficient implementation, fine tuning, exploiting structure of problem and instances and experimenting on an (randomly generated) extensive testbed, showed a very limited success, if any at all.

A direct application of integer programming solvers is not a practical and 
realistic approach as for even very small size instances, no feasible solution was reported in a reasonable time limit. Moreover, the model is a non-convex one for which, to the best of our knowledge, no solver can provide a provably optimal solution. Similar arguments apply when developing decomposition approaches based on the idea of (Generalized) Benders decomposition.

\subsection{Local search}

A practical approach, which is still limited in size, is a combination of a simple local search, which explores the space of some discrete variables (particularly, the binary variables $\eta_{k l}^{v s o}$ ) and solve the reduced problems (obtained by fixing the chosen discrete variables in the original problem COOPTV-HLP). In the sequel, we refer to this reduced problem by COOPTV-HLP $\left(\bar{\eta}_{k l}^{v s o}\right)$ that turns to be convex and can be solved using a nonlinear programming solver.

Note that once the binary variables $\bar{\eta}_{k l}^{v s o}, \forall k, l, v, s, o$ are fixed, the speed, the vessel class and the connection to be opened become known. The consequences are the following:

(1) the transportation cost term in the objective function becomes a linear term:

$$
\begin{aligned}
& \sum_{o \in \mathcal{O}}\left(\sum_{i, j \neq i} R_{i j}^{o} \delta_{i j}^{o}-\left[\sum_{k, l \neq k} \alpha^{o} C_{k l}^{o} x_{i j k l}^{o}+\sum_{(k, l) \in \mathcal{N}_{o} \times \mathcal{N}_{\bar{o}}, k \neq l} \alpha_{k l}^{v s o} C_{k l}^{o} \bar{\eta}_{k l}^{v s o} x_{i j k l}^{o}+\right.\right. \\
& \left.\left.\sum_{k \neq i, j} C_{i k}^{o} a_{i j k}^{o}+\sum_{k \neq i, j} C_{k j}^{o} b_{i j k}^{o}+C_{i j}^{o} e_{i j}^{o}\right]\right)
\end{aligned}
$$

(2) constraints (19) become linear,

(3) the set of links connecting two operators, i.e. $y_{k l}$ variables (not $y_{k l}^{o}$ ), will be completely characterized and therefore some of the variables $z_{k l}^{-o}$ in constraints (26) will become fixed and for $y_{k l}^{o}=0$, constraints (4), (5), and (24) will be eliminated,

(4) constraints (25) help in filtering solutions in the neighborhood and avoid evaluating objective function for infeasible solutions, e.g. combination of transporters exceeding the capacity of jointly installed edges. This can significantly reduce the neighborhood size.

The neighborhood structure is composed of solutions that are different from the current solution with respect to 1) the pairs of nodes chosen to be established $\left.\left(\left|\mathcal{N}_{\mathcal{A}}\right|\left|\mathcal{N}_{\mathcal{B}}\right|\right), 2\right)$ the vessel classes to be deployed on that edge $(|\mathcal{V}|)$, and 3) the sailing speed $(|\mathcal{S}|)$.

An efficient local search is developed to explore a large neighborhood $\mathcal{N}$ with the cardinality of $\left|\mathcal{N}_{\mathcal{A}}\right|\left|\mathcal{N}_{\mathcal{B}}\right||\mathcal{V}||\mathcal{S}|$. In practice, the real size of such a neighborhood is by far smaller than $\left|\mathcal{N}_{\mathcal{A}}\right|\left|\mathcal{N}_{\mathcal{B}}\right||\mathcal{V}||\mathcal{S}|$ due to several reasons.

The first reason is that the number of cooperatively established edges is much less than $\left|\mathcal{N}_{\mathcal{A}}\right|\left|\mathcal{N}_{\mathcal{B}}\right|$ because some links connecting two extreme points of different operators can only appear in an optimal solution with negligible probability (see e.g. an edge that may connect nodes \#5 and \#17). Even if they can theoretically 
appear in an optimal solution, there are several practical (political and economic) reasons to convince practitioners that this is not at all acceptable from the industry point of view as it can threaten and jeopardize the whole structure of every operator's network by introducing revolutionary changes causing different concerns such as creating tensions, introducing some cannibalization effects or threatening strategic interests of the mother company from among others. This is not much welcome from the practitioners' side. Similar arguments apply to the very short connections. They cannot appear neither, as the port call costs (including hotel costs, i.e. the labor fees while vessel is standstill at a port for being unloaded) are non-negligible when compared to the revenue and the economies of scale cannot be exploited.

The second reason is that not every vessel type suits a given edge that is operated as a pendulum service. Therefore, often the choices are strictly less than $|\mathcal{V}|$

The third reason is again related to the vessel. The fleet runners have a very good knowledge of their fleet elements. As almost $50 \%$ of the transportation cost is incurred by the bunker cost, practitioners have a very clear idea that by no extent a given vessel $v$ of class $\mathcal{V}$ may be at all operated at the given speed. In addition to this, one can also take into account speed limits dictated by some authorities within some territorial water that restricts the choices for an economic shipping.

However, the computational tractability of the resulting reduced (parameterized) version of model is still limited due to three major issues: 1) the Big-M nature of the model arising from constraints $(19), 2)$ nonlinearity in the $f_{k, l}^{+o}\left(z_{k l}^{-}\right)$, and 3 ) the limitation of the state-of-the-arts solvers when facing such large size nonlinear problems. This suggests that trajectory-based (or single solution heuristics) are preferred to the population-based heuristics as we will not be able to efficiently evaluate, in each iteration, the objective function for even a small size population.

\subsection{Lagrangian relaxation}

Evaluating a candidate solution in the neighborhood of the current solution in our heuristic corresponds to solving a MIP problem (COOPTV-HLP $\left(\bar{\eta}_{k l}^{v s o}\right)$ ), which is highly intractable and is the bottleneck for the efficiency of our heuristic. While a direct application of a generic MIP solver is not an efficient way to solve this problem (and no other efficient tailored algorithm is available), a similar problem is studied in [15] wherein the authors proposed a Lagrangian relaxation-based algorithm to evaluate it. That relaxation can be adapted to our parameterized problem, as well. The major difference is that in the case of COOPTV-HLP $\left(\bar{\eta}_{k l}^{v s o}\right)$, a quadratic term appears in the objective function that includes continuous variables.

We choose to relax the constraints (8)-(11), (14)-(18), (20)-(22) using Lagrangian multipliers with appropriate sizes and signs.

By doing so, the Lagrangian relaxation problem is decomposable into two subproblems: 1) the space of flow variables, and 2) other variables including network design variables. 
The first subproblem shows some behaviors very similar to the integrality property. Often the resulting solutions are integer, even if it is solved as a simple LP. The second problem is a quadratic one, which is relatively easy to solve using a nonlinear solver such as Baron or even CPLEX.

\subsection{The algorithm}

There are some differences when compared to the algorithm presented in [15]. The major difference is that instead of the well-known subgradient method, we use bundle method of Frangioni [12] for solving the Lagrangian dual problem, which significantly accelerated the convergence and reduced the number of iterations as the step sizes are chosen with higher precision. Furthermore, We start with iterations of a continuous relaxation of COOPTV-HLP $\left(\bar{\eta}_{k l}^{v s o}\right)$ for a given time limit per iteration and once sufficient number of iterations is reached, we switch to the COOPTV-HLP $\left(\bar{\eta}_{k l}^{v s o}\right)$ with its binary variables.

We have three dimensions to search in our neighborhood, i.e. cooperative edges to establish, vessel classes to choose and sailing speed to determine. We start with the cooperative edges, a lower priority to vessel class and then the lowest priority to the sailing speed determination. The logical argument is that first the operating edges must be determined and the potential volume of flow on the edges must be estimated before the vessel type can be determined. Furthermore, the sailing speed choices depend strongly on the vessel class where expert's knowledge can play an important role.

Later in the process, one tries to improve the solution by taking into account different combination of speed and vessel classes based on the priorities identified by the experts.

We terminate the search if any of the following conditions is satisfied: 1) reaching a maximum iteration number, 2) some iterations with no improvement, and 3) gap drops below a threshold.

A general framework of our algorithm is presented in Algorithm 1. In this algorithm gUB and gLB represent the global upper and global lower bounds, respectively. 


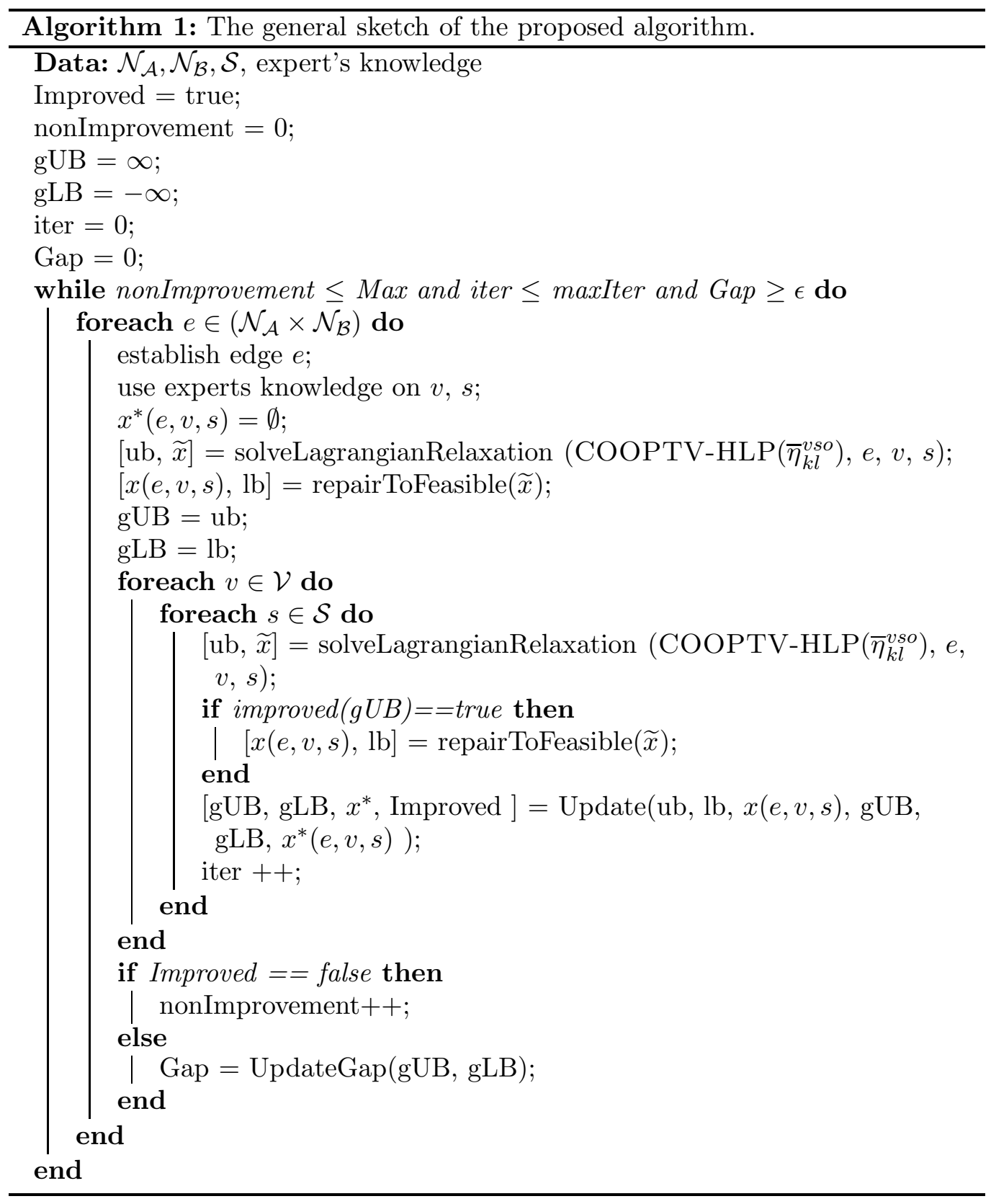

\section{Numerical Experiments}

Computational experiments with real data obtained from the two sister companies both belonging to one of the major operators are carried out on a case study from Asia. Any explanation about the choice of parameters and expert's knowledge reveals part of an information that is highly sensitive and violates the confidentiality agreements from the perspective of our industrial partner. However, we are still able to elaborate on the statistics collected in the course of our numerical experiments.

Our real case is composed of 60 nodes in total. In order to obtain smaller size instances (e.g. 20, 30 etc.) we made some aggregations enabling us to create 
artificial coordinates with concentrated flows. The data, numbers and figures are linearly scaled such that while respecting the confidentiality, the structure of data is preserved.

We set a time limit of $25\left|\mathcal{N}_{\mathcal{A}}+\mathcal{N}_{\mathcal{B}}\right|$ seconds for every call to the bundle method. The termination criteria are set to the number of non-improving bounds (lower and upper), a maximum number of iterations and a time limit of 120 hours (slight deviation from this value is possible because sometimes the last iteration of bundle method could not be stopped at the exact time). The computational experiments are carried out on a Processor Intel(R) Core(TM) i7-4770 CPU @ 3.40GHz, 4 Core(s), 8 Processor(s) and 16,0 GB of RAM.

The role of Lagrangian relaxation is to report an approximation of the objective function rather than computing it exactly. That is, to identify the links to open and the vessel class to deploy, with a good approximation. However, we do not expect that the reported sailing speed be very precise. It suffices and is acceptable from the practical viewpoint, because speed is a kind of tactical/operational decision that can be adjusted later on and does not play a major role in the longer term decisions such as the edges to open and the types of vessels to deploy. Therefore, we do not intend to spend too many iterations of bundle method with very marginal improvements.

Each time the upper bound is improved, we use the information of Lagrangian relaxation (the dual multipliers etc.) to repair and restore a feasible solution. The best solution is then accepted as the best-found lower bound.

Table 3 outlines a part of our numerical results on our testbed. The table contains the results on 20 instances in five block rows corresponding to different sizes $\left|\mathcal{N}_{\mathcal{A}}\right|+\left|\mathcal{N}_{\mathcal{B}}\right| \in\{20,30,40,50,60\}$ each of which contains 4 instances for different combinations of $\left|\mathcal{N}_{\mathcal{A}}\right|$ and $\left|\mathcal{N}_{\mathcal{B}}\right|$.

CPLEX 12.6.2 is used to solve the MIP problems in the iterations of bundle and the relative gap is set to some values between 0.5 to 10 percent (depending on the size of instance) as often such solutions are indeed optimal but CPLEX needs long time to prove the optimality. 
Table 3.: Numerical experiments

\begin{tabular}{|c|c|c|c|c|c|c|}
\hline instance name & $\left|\mathcal{N}_{\mathcal{A}}\right|+\left|\mathcal{N}_{\mathcal{B}}\right|$ & best upper bound & best lower bound & $\operatorname{Gap}(\%)$ & Total Time (sec.) & additional market (\%) \\
\hline $\begin{array}{l}\text { LS1-1 } \\
\text { LS1-2 } \\
\text { LS1-3 } \\
\text { LS1-4 }\end{array}$ & $\begin{array}{l}10+10 \\
10+10 \\
10+10 \\
10+10\end{array}$ & $\begin{array}{l}183,159.90 \\
167,088.92 \\
159,466.65 \\
202,362.60\end{array}$ & $\begin{array}{l}178,283.61 \\
158,758.95 \\
158,984.11 \\
197,281.35\end{array}$ & $\begin{array}{l}2.66 \\
5.24 \\
0.30 \\
2.58\end{array}$ & $\begin{array}{l}4,717.34 \\
4,400.73 \\
5,244.94 \\
3,772.57\end{array}$ & $\begin{array}{l}12.38 \\
14.81 \\
13.78 \\
14.14\end{array}$ \\
\hline $\begin{array}{l}\text { LS2-1 } \\
\text { LS2-2 } \\
\text { LS2-3 } \\
\text { LS2-4 }\end{array}$ & $\begin{array}{l}15+15 \\
15+15 \\
10+20 \\
20+10\end{array}$ & $\begin{array}{l}199,413.55 \\
253,150.76 \\
208,795.65 \\
193,510.93\end{array}$ & $\begin{array}{l}172,635.75 \\
224,734.65 \\
176,130.29 \\
173,965.05\end{array}$ & $\begin{array}{l}15.51 \\
12.64 \\
15.64 \\
11.23\end{array}$ & $\begin{array}{c}9,982.54 \\
10,010.37 \\
9,990.61 \\
9,258.43\end{array}$ & $\begin{array}{l}13.30 \\
12.69 \\
12.76 \\
11.33\end{array}$ \\
\hline $\begin{array}{l}\text { LS3-1 } \\
\text { LS3-2 } \\
\text { LS3-3 } \\
\text { LS3-4 } \\
\end{array}$ & $\begin{array}{l}20+20 \\
20+20 \\
10+30 \\
15+25\end{array}$ & $\begin{array}{l}302,784.32 \\
319,669.96 \\
294,959.53 \\
293,813.39\end{array}$ & $\begin{array}{l}245,184.50 \\
268,586.85 \\
242,632.95 \\
237,637.05\end{array}$ & $\begin{array}{l}19.02 \\
19.01 \\
21.56 \\
23.63\end{array}$ & $\begin{array}{l}14,201.76 \\
17,177.26 \\
17,788.80 \\
13,709.03\end{array}$ & $\begin{array}{l}11.78 \\
11.03 \\
14.31 \\
12.53\end{array}$ \\
\hline $\begin{array}{l}\text { LS5-1 } \\
\text { LS5-2 } \\
\text { LS5-3 } \\
\text { LS5-4 }\end{array}$ & $\begin{array}{l}25+25 \\
20+30 \\
15+35 \\
10+40\end{array}$ & $\begin{array}{l}374,115.52 \\
352,317.91 \\
378,934.60 \\
375,496.00\end{array}$ & $\begin{array}{l}295,963.55 \\
281,607.92 \\
294,329.70 \\
293,546.48\end{array}$ & $\begin{array}{l}26.40 \\
25.10 \\
28.74 \\
27.91\end{array}$ & $\begin{array}{l}27,141.23 \\
25,165.81 \\
21,881.69 \\
24,963.07\end{array}$ & $\begin{array}{l}11.54 \\
12.55 \\
13.43 \\
12.87\end{array}$ \\
\hline $\begin{array}{l}\text { LS4-1 } \\
\text { LS4-2 } \\
\text { LS4-3 } \\
\text { LS4-4 }\end{array}$ & $\begin{array}{l}30+30 \\
25+35 \\
20+40 \\
15+45\end{array}$ & $\begin{array}{l}482,394.83 \\
479,538.49 \\
493,598.64 \\
492,536.00\end{array}$ & $\begin{array}{l}367,816.05 \\
367,972.52 \\
378,360.15 \\
369,249.36\end{array}$ & $\begin{array}{l}31.15 \\
30.31 \\
30.45 \\
33.38\end{array}$ & $\begin{array}{l}395,769.24 \\
338,502.40 \\
402,245.63 \\
439,905.24\end{array}$ & $\begin{array}{l}12.03 \\
13.82 \\
12.78 \\
11.87\end{array}$ \\
\hline
\end{tabular}


In Table 3, the first column represents the name of instance and the second column represents the size of network (the number of nodes). The best upper and lower bounds and the related gap are reported in the next columns. The column 'Total Time (sec.)' reports the total time spent before termination of algorithm. In column 'additional market' we report the percentage of additional demand generated as a results of such co-opetition.

One observes in Table 3 that as the size of instance increases, the computational time increases in a nonlinear manner. This is mainly due to the evaluation of the MIP model using Lagrangian relaxation method. While in the smaller instances, many bundle calls can be made within a relatively short time, this becomes a bottleneck in the larger sizes. For the same reasons the quality of solutions deteriorate as the instances get larger. Moreover for the same $\left|\mathcal{N}_{\mathcal{A}}+\mathcal{N}_{\mathcal{B}}\right|$, as $\left|\mathcal{N}_{\mathcal{A}}-\mathcal{N}_{\mathcal{B}}\right|$ increases the gap tends to increase. The method evidently works better when the operations are of more similar sizes.

In terms of additional volume of market share generated relative to the actual aggregated volume of market shares of both operators, we see that this value is almost always between 10 and 15 percent. Given that our method is essentially a heuristic method finding a suboptimal (no proven optimality), this percentage is a lower bound on the actual added value (calculated based on optimal solution) of the co-opetition. On the other hand, as all the 20 instances are generated from the same base instance of 60 nodes, it is normal that this value remains almost the same in all the instances. It must be noted that the share of each operator from this additional market share is proportional to its level of competition in the best-found solution.

\section{Conclusion and Future Work}

We proposed one of the first co-opetitive network design and fleet deployment problems, which is formulated as a mono-level and mono-objective mathematical programming formulation. The model is a generic model that we customized it to take into account features from the liner shipping industry. The resulting model is a large scale optimization problem. Evidently, this model is highly intractable even for very small size instances. To solve this model, we proposed a matheuristic solution algorithm integrating Lagrangian relaxation within a local search algorithm that can be used to obtain good solutions and acceptable from the industrial point of view.

In future work, we establish further theoretical grounds for our framework proposed for co-opetition. One may in addition be interested to identify the relationship with relevant topics in the literature. Extension to other domains of application such as scheduling, routing, resource allocations etc. arising in logistics, supply chain and telecommunications -wherein several operators are sharing the infrastructure- also deserve attention. The major issue we encountered here is twofold: the non-convexity of our mathematical model and the size of resulting model. In order to deal with the first issue one can consider joint inner/outer approximation techniques (or perhaps some local convexification techniques). Alternative mathematical models also deserve further attention. Given the particular O-D flow sub-structure, one can also consider some sort of parallelization of solution process, which is of interest both in exact approaches as well as in matheuristics 
and metaheuristics. We particularly focus on combining matheuristics with primal and dual decomposition techniques where by fixing a part of the decisions, the remaining problem has a convex linear relaxation. We also work on generating a public domain testbed based on some perturbed/scaled/skewed data from the real practice.

\section{Acknowledgments}

This work is supported by the PGMO, the Gaspard Monge Program for Optimisation and Operational Research in the framework of BENMIP project and by the Campus interdisciplinaire de recherche, d'innovation technologique et de formation Internationale sur la Sécurité et l'Intermodalité des Transports (CISIT) —ELSAT project. The authors are thankful of the anonymous referees for their constructive comments that significantly improved the article.

\section{References}

[1] Adler, N., 2001. Competition in a deregulated air transportation market. European Journal of Operational Research 129 (2), 337-345.

[2] Adler, N., Smilowitz, K., 2007. Hub-and-spoke network alliances and mergers: Pricelocation competition in the airline industry. Transportation Research Part B: Methodological 41 (4), $394-409$.

[3] Alumur, S., Kara, B. Y., 2008. Network hub location problems: the state of the art. European Journal of Operational Research 190, 1-21.

[4] Brandenburger, A., Nalebuff, B., 1996. La co-opétition, une révolution dans la manière de jouer concurrence et coopération. Village Mondial.

[5] Brandenburger, A. M., Nalebuff, B. J., 2011. Co-opetition. Crown Business.

[6] Campbell, J. F., Ernst, A. T., Krishnamoorthy, M., 2002. Hub location problems. In: Drezner, Z., Hamacher, H. W. (Eds.), Facility Location: Applications and Theory. Springer, pp. 373-407.

[7] Campbell, J. F., O'Kelly, M. E., 2012. Twenty-five years of hub location research. Transportation Science 46 (2), 153-169.

[8] Čvokić, D. D., Kochetov, Y. A., Plyasunov, A. V., 2016. A leader-follower hub location problem under fixed markups. In: International Conference on Discrete Optimization and Operations Research. Springer, pp. 350-363.

[9] De Ngo, D., Okura, M., 2008. Coopetition in a mixed duopoly market. Economics Bulletin $12(20), 1-9$.

[10] Dyer, J. H., Singh, H., 1998. The relational view: Cooperative strategy and sources of interorganizational competitive advantage. Academy of management review 23 (4), 660-679.

[11] Eiselt, H., Marianov, V., 2009. A conditional p-hub location problem with attraction functions. Computers \& Operations Research 36 (12), 3128 - 3135.

[12] Frangioni, A., 1996. Solving semidefinite quadratic problems within nonsmooth optimization algorithms. Computers \& Operations Research 23 (11), 1099-1118.

[13] Gelareh, S., Nickel, S., 2011. Hub location problems in transportation networks. Transportation Research Part E: Logistics and Transportation Review 47 (6), 1092 - 1111.

[14] Gelareh, S., Nickel, S., Pisinger, D., 2010. Liner shipping hub network design in a competitive environment. Transportation Research Part E: Logistics and Transportation Review 46 (6), 991 - 1004.

[15] Gelareh, S., Nickel, S., Pisinger, D., 2010. Liner shipping hub network design in a competitive environment. Transportation Research Part E: Logistics and Transportation Review 46 (6), 991-1004. 
[16] Geoffrion, A. M., 1972. Generalized benders decomposition. Journal of optimization theory and applications 10 (4), 237-260.

[17] Gnyawali, D. R., Park, B.-J. R., 2011. Co-opetition between giants: Collaboration with competitors for technological innovation. Research Policy 40 (5), 650-663.

[18] Gutiérrez-Jarpa, G., Laporte, G., Marianov, V., Moccia, L., 2017. Multi-objective rapid transit network design with modal competition: The case of concepción, chile. Computers \& Operations Research 78, 27-43.

[19] Kara, B. Y., Taner, M. R., 2011. Hub location problems: The location of interacting facilities. In: Eiselt, H. A., Marianov, V. (Eds.), Foundations of location analysis. Springer, pp. 273-288.

[20] Li, Y., Liu, Y., Liu, H., 2011. Co-opetition, distributor's entrepreneurial orientation and manufacturer's knowledge acquisition: Evidence from china. Journal of Operations Management 29 (12), 128 - 142, special Issue Operations management, entrepreneurship, and value creation: Emerging opportunities in a cross-disciplinary context.

[21] Lin, D.-Y., Huang, C.-C., 2013. Coopetition in international maritime shipping. Polish Maritime Research 20 (Special Issue), 5-12.

[22] Lüer-Villagra, A., Marianov, V., 2013. A competitive hub location and pricing problem. European Journal of Operational Research 231 (3), 734-744.

[23] Mahmutogullari, A. I., Kara, B. Y., 2016. Hub location under competition. European Journal of Operational Research 250 (1), 214-225.

[24] Marianov, V., Serra, D., ReVelle, C., 1999. Location of hubs in a competitive environment. European Journal of Operational Research 114 (2), 363-371.

[25] Martın, J. C., Román, C., 2004. Analyzing competition for hub location in intercontinental aviation markets. Transportation Research Part E: Logistics and Transportation Review 40 (2), 135-150.

[26] Notteboom, T., Carriou, P., April 2009. Fuel surcharge practices of container shipping lines: Is it about cost recovery or revenue making? In: Proceedings of the 2009 International Association of Maritime Economists (IAME) Conference. Copenhagen, Denmark.

[27] Sasaki, M., 2005. Hub network design model in a competitive environment with flow threshold. Journal of the Operations Research Society of Japan 48 (2), 158-171.

[28] Sasaki, M., Campbell, J. F., Krishnamoorthy, M., Ernst, A. T., 2014. A stackelberg hub arc location model for a competitive environment. Computers \& Operations Research 47, 27-41.

[29] Sasaki, M., Fukushima, M., December 2001. Stackelberg hub location problem. Journal of the Operations Research Society of Japan 44 (4), 390-405.

[30] Smith, K. G., Grimm, C. M., Gannon, M. J., 1992. Dynamics of competitive strategy. Sage Publications, Inc.

[31] Stopford, M., 2009. Maritime Economics 3e. Routledge.

[32] Yami, S., Castaldo, S., Dagnino, B., Le Roy, F., 2010. Coopetition: winning strategies for the 21st century. Edward Elgar Publishing.

[33] Zhang, J., Frazier, G. V., 2011. Strategic alliance via co-opetition: Supply chain partnership with a competitor. Decision Support Systems 51 (4), 853 - 863, recent Advances in Data, Text, and Media Mining; Information Issues in Supply Chain and in Service System Design. 